\title{
Biomechanical Evaluation of a Patient-Handling Technology Prototype
}

\author{
Hardianto Iridiastadi ${ }^{*}$, Theodora Vani ${ }^{1}$, Putra Alif Ramdhani Yamin ${ }^{1}$ \\ ${ }^{1}$ Industrial Engineering, Faculty of Industrial Technology, Institut Teknologi Bandung (ITB), Indonesia, Ganesa \\ 10, Bandung 40132, Indonesia
}

\begin{abstract}
Nursing jobs have generally been associated with poor posture and heavy exertion, particularly during patient-handling activities. Such working conditions are known to increase the risk of musculoskeletal disorders, and the use of patient-handling aids has been proposed to alleviate the problem. This study was conducted with the purpose of evaluating a patient-handling technology (PHT) prototype that was developed by the researchers for use in Indonesia. An experiment was conducted involving 12 nurse participants, who were asked to move a patient, either manually or by using the prototype. Postural analyses were performed using 3DSSPP software, Rapid Upper Limb Assessment (RULA), and Rapid Entire Body Assessment (REBA). A Borg scale was employed to determine the participants' perceptions of the levels of exertion required during the experiment. The findings demonstrated that using the PHT prototype resulted in a substantial reduction in the compression force at the lumbar (L5/S1) joint. However, there was a marginal increase in shear force at this joint due to the push/pull activities involved in using the technology while handling the patient. This increase, however, was still within accepted biomechanical limits. Postural analyses based on the RULA and REBA demonstrated a great reduction in ergonomic risks when using the prototype. The nurses also reported reduced physical workload when using the PHT technology. Across all measures, there were reductions in the biomechanical risks of between $30 \%$ and nearly $70 \%$. While the PHT prototype still needs design refinements, its use in medical settings has the potential to ameliorate musculoskeletal problems among nurses and caregivers.
\end{abstract}

Keywords: Caregivers; Musculoskeletal disorders; Patient handling; Patient-handling technology prototype;

\section{Introduction}

The patient-handling activities performed by hospital nurses are often associated with increased risk of musculoskeletal disorders or MSDs (Waters et al., 2007; Nelson et al., 2007; Iridiastadi et al., 2019). These are a group of disorders affecting the muscles, joints, tendons, ligaments, nerves, and bones, which are caused by strenuous physical activity in combination with poor posture (Batubara and Dharmastiti, 2017; Ribeiro et al., 2017). According to the US Bureau of Labor Statistics (2008), caregivers are subject to a relatively high rate of non-fatal occupational injuries. Caregivers include nurses, health workers, and nursing assistants. Nursing has been identified as a profession carrying a high risk of lower back or spinal injury. Every year, 10,000 nurses in the United States suffer MSDs and roughly $30-65 \%$ of these injuries are caused by manual patient-handling activities. 
These activities include positioning a patient manually, moving a patient between a bed and a chair, helping a patient to move around, and helping a patient to rise from a sitting position (Hodder et al., 2010).

One of the methods for reducing the risk of MSDs is to use lifting or moving aids (Nelson et al., 2006). In the United States, for instance, many medical settings have introduced patient-handling technology to reduce the risk of musculoskeletal injury. Hospitals employ floor lifts and ceiling lifts with patients. The floor lift is a portable tool that can be maneuvered on the floor so that, when handling a patient, the nurse only needs to push and control the device. The ceiling lift is usually installed on the ceiling of the room, and the nurse can move the patient by using a controller or a remote control.

Although these ideas are not new in many industrialized countries, the concept and availability of patient-handling aids is generally lacking in many developing nations. In Indonesia, such apparatus is generally only available in the larger hospitals, or it is available for rent at a high price. In recent years, the researchers have developed a patient-handling technology (PHT) prototype in the expectation that this will prove to be low-cost technology that will help reduce the risk of MSDs in Indonesian nurses and caregivers. This prototype can be used to help maneuver a patient from a bed to a wheelchair and vice versa. Two types of lifting mat are available-for the sitting and supine positions. The tool is activated through a pneumatic system; and the nurse or caregiver controls the height and position of the tool through a remote control. The PHT prototype is also equipped with wheels and a braking pedal.

Despite its potential benefits, the prototype had not yet been evaluated from the biomechanical perspective. Its effectiveness had not been proven, and it was not clear if nurses would accept the technology in the hospital setting. The major research question was whether the technology would result in lower biomechanical loads and thus, potentially, reduce the risk of MSDs. Even if it did result in lower loads, there was also a need to determine whether the loads were indeed below the acceptable ergonomic limit. If the technology was accepted by the users, and shown to perform as intended, the prototype could be manufactured and made widely available in a large number of hospitals in Indonesia.

\section{Methods}

In this study, an experiment was conducted with the purpose of testing the PHT prototype already developed. Nurse participants were asked to move patients from a bed to a wheelchair and back, either manually or by using the PHT prototype. Postural analyses of the two approaches were conducted, and statistical comparisons of several key postures were made. The approach followed that proposed by Dutta et al. (2011a) and Alamgir et al. (2008). Following our previous study, differences in patient anthropometric data were incorporated into the prototype design. Specifically, the design of the PHT lifting mat was adjusted according to Indonesian anthropometric data.

\subsection{Participants}

In this experiment, a male (weighing $80 \mathrm{~kg}$ ) acted as the patient. For the purposes of the experiment, the patient was described as an individual with partial infirmity, enabling him to still place a foot on the floor during the maneuvering activities. Twelve female nurses participated, who ranged in age from 20 to 50 years. This number of participants was considered acceptable for this preliminary investigation as the statistical power was greater than 0.8 (i.e., it correctly rejected the null hypothesis). The nurses had all worked at a hospital for at least one year, and reported no current muscular or skeletal injury. The order of the handling conditions was randomly determined (McGuigan, 1990), with the 
expectation that the results would not be influenced by the participants' learning process. The minimum number of participants was calculated based on the effect size " $d$ " as proposed by Maxwell and Delaney (2004).

\subsection{Tools and Equipment}

The tools and apparatus in this experiment included a PHT prototype, a patient bed, a wheelchair, and a camera that recorded the nurses' postures from two different angles. The experiment was carried out in a room that was set up to simulate a patient's room in a hospital (Figure 1).

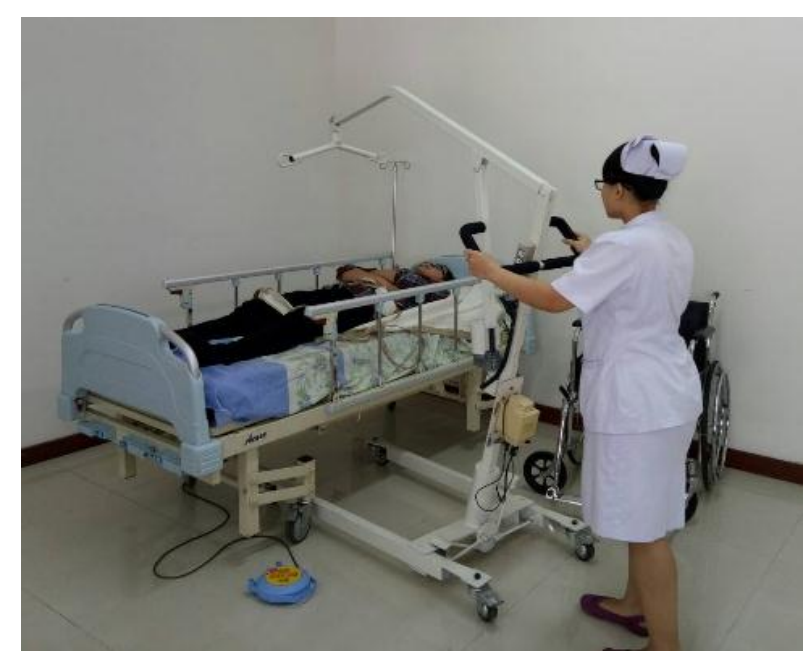

Figure 1 Experimental setting for the study

\subsection{Procedures}

The nurse participants were asked to perform a patient handling simulation from a bed to a wheelchair and back, either manually (as they would normally do in a hospital setting) or by using the PHT prototype (with procedures according to Dutta et al., 2011b). The experimental design was within subjects in that each nurse participant performed the same experiment. A training session was given prior to the actual experiment. A digital camera was utilized for capturing changes in body posture adopted during the simulation. Markers were attached to body joints for the purpose of determining joint angles. The manual condition consisted of five key postures (Table 1): (1) the nurse supports the upper body of the patient; (2) the nurse lowers the patient's legs from the bed; (3) the nurse positions her body; (4) the nurse lifts and moves the patient; and (5) the nurse positions the patient in the wheelchair.

Differing from the manual condition, the simulation with the PHT prototype consisted of seven major postures (Table 2), as described by Dutta et al. (2011b): (1) the nurse puts a mat under the patient on the bed; (2) the nurse presses the button on the PHT to lift the patient; (3) the nurse pulls the PHT; (4) the nurse rotates and guides the PHT toward the wheelchair; (5) the nurse pushes the PHT; (6) the nurse presses the button to lower the patient, and lastly; (7) the nurse removes the mat from the wheelchair. 
Table 1 The five postures in the manual condition

\begin{tabular}{ccccc}
\hline Posture 1 & Posture 2 & Posture 3 & Posture 4 & Posture 5 \\
\hline & & & \\
& & &
\end{tabular}

Table 2 The seven postures adopted in the PHT prototype condition

\begin{tabular}{cccc}
\hline Posture 1 Posture 2 & Posture 3 & Posture 4 \\
\hline &
\end{tabular}

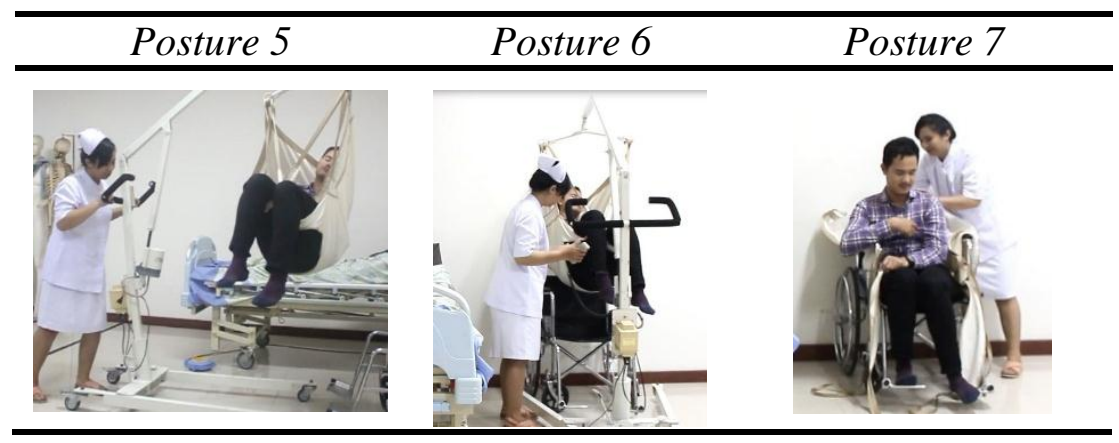

\subsection{Data Analysis}

The compression and shear force exerted at the L5/S1 joint were calculated using the 3 Dimension Static Strength Prediction Program (3DSSPP) developed by the University of Michigan, Ann Arbor, MI, USA. This software was used to quantitatively model the force and movement at each body joint based on the posture adopted during the maneuver and the external force (the mass of the patient) acting on the hand/arm/shoulder area. The calculated compression and shear force at the lumbar (L5/S1) joint, in particular, were compared against accepted biomechanical criteria. The anthropometric data of the nurses were measured using an anthropometer (Yuhaniz et al., 2017), and the resulting joint angles at each stage of the handling task were the major inputs for the software. The postures adopted by the nurses during the simulated patient-handling tasks were also quantified by using the Rapid Entire Body Assessment (Hignett and McAtamney, 2000) and the Rapid Upper Limb Assessment, RULA (McAtamney \& Corlett, 1993). The nurses' perceptions regarding the amount of force they exerted during the simulation were quantified using the Borg CR-10 scale (Borg, 1990). 


\subsection{Statistical Analysis}

Each value for the compression force and shear force were tested statistically by the paired t test to look for significant differences in the two force value of similar postures, and the ANOVA $(\mathrm{p}<0.05)$ was used to see the significance of each posture group. The statistical testing was done with the Minitab software version 17. The values obtained from nurses in similar postures were not significantly different, so they could be averaged. For the REBA and RULA values, a non-parametric test was conducted to look for significant differences in each group of data. The significance was then tested with ANOVA $(p<0.05)$. The values for each key posture were found to be not significantly different so the values for each key posture could be averaged.

\section{Results}

In total, there were 288 postures (one simulation cycle with 12 nurses) to be modeled in 3DSSPP. These were further analyzed using REBA and RULA. All the Borg ratings are described in the following section.

\subsection{Compression and Shear Forces}

The posture modeling method using the 3DSSPP software resulted in compression forces and shear forces being identified for each posture. The major inputs included the nurses' anthropometric data, joint angles, and the loads received during each posture. Figure 2 demonstrates an example of the posture of a nurse as modeled in 3DSSPP.
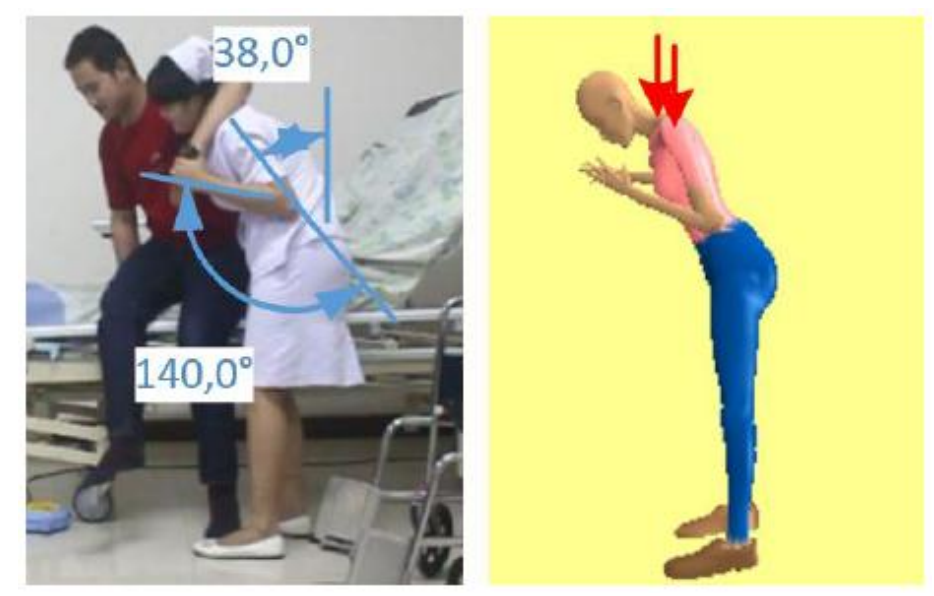

Figure 2 Posture of a participant during simulated patient handling

After obtaining the compression force data from all nurses and for all postures, a statistical test was conducted to examine differences in distribution. Based on paired t-tests, each group of similar posture compression data values yielded $p>0.05$, meaning that there were no significant differences between similar postures. The two groups of data were thus averaged. Analysis of Variance (ANOVA) tests were conducted for all key postures, and resulted in $\mathrm{p}<0.05$, implying that the means across key postures were significantly different. This statistical analysis was also applied to shear force values.

Figure 3 shows the average compression force for each of the five (manual handling) and seven (handling with PHT) key postures. It can be seen that the manual handling of the patients resulted in compression forces ranging from 1,000 to 1,800 N. The greatest compression force occurred during positioning the patient from or on the wheelchair. In contrast, using the PHT prototype, these values ranged from roughly 200 to $1,100 \mathrm{~N}$ (a 
reduction in maximum force of nearly 40\%). For the prototype, the largest compression forces occurred while the nurse pushed or pulled the PHT.

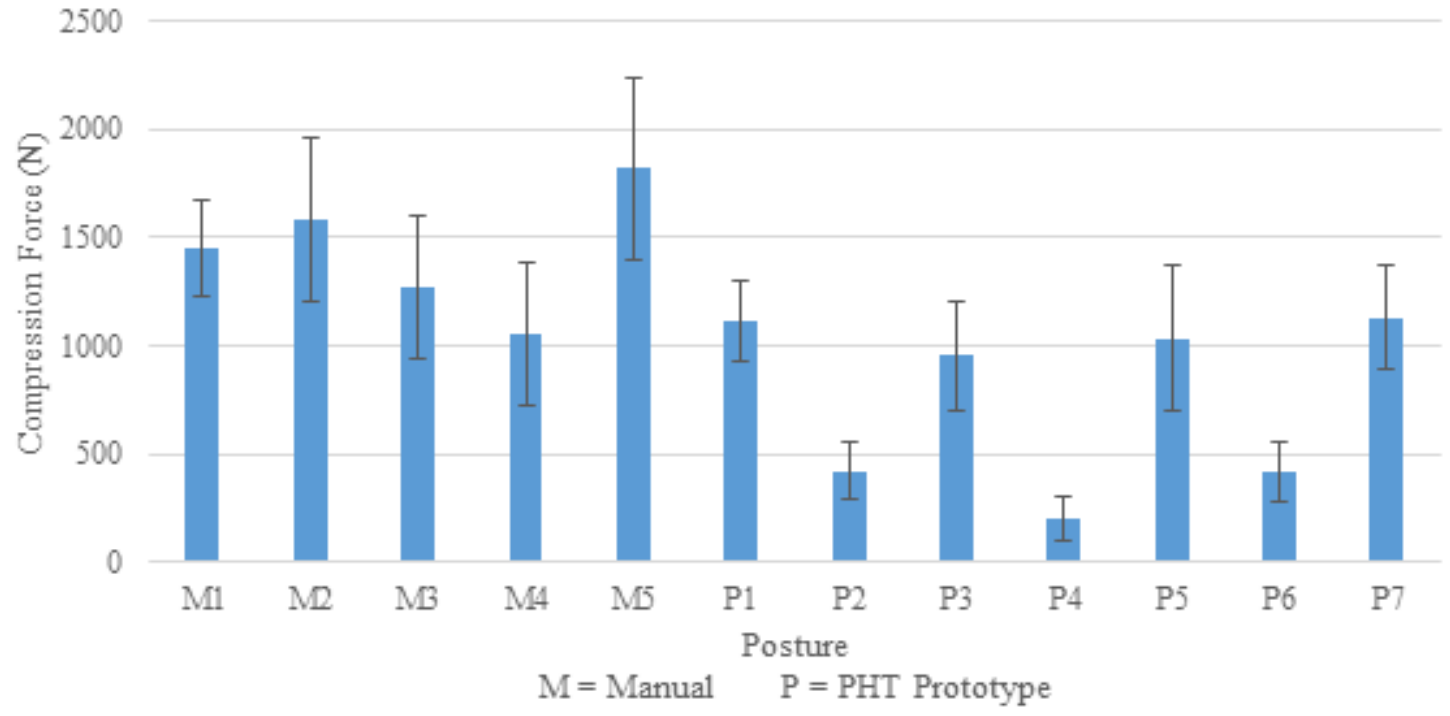

Figure 3 Compression force (mean and standard deviation)

The shear force during manual handling ranged from around 200 to $250 \mathrm{~N}$, while the corresponding values when using the prototype were between 50 and $360 \mathrm{~N}$. The data indicated that the use of the PHT did result in greater shear force at the L5/S1 joint.

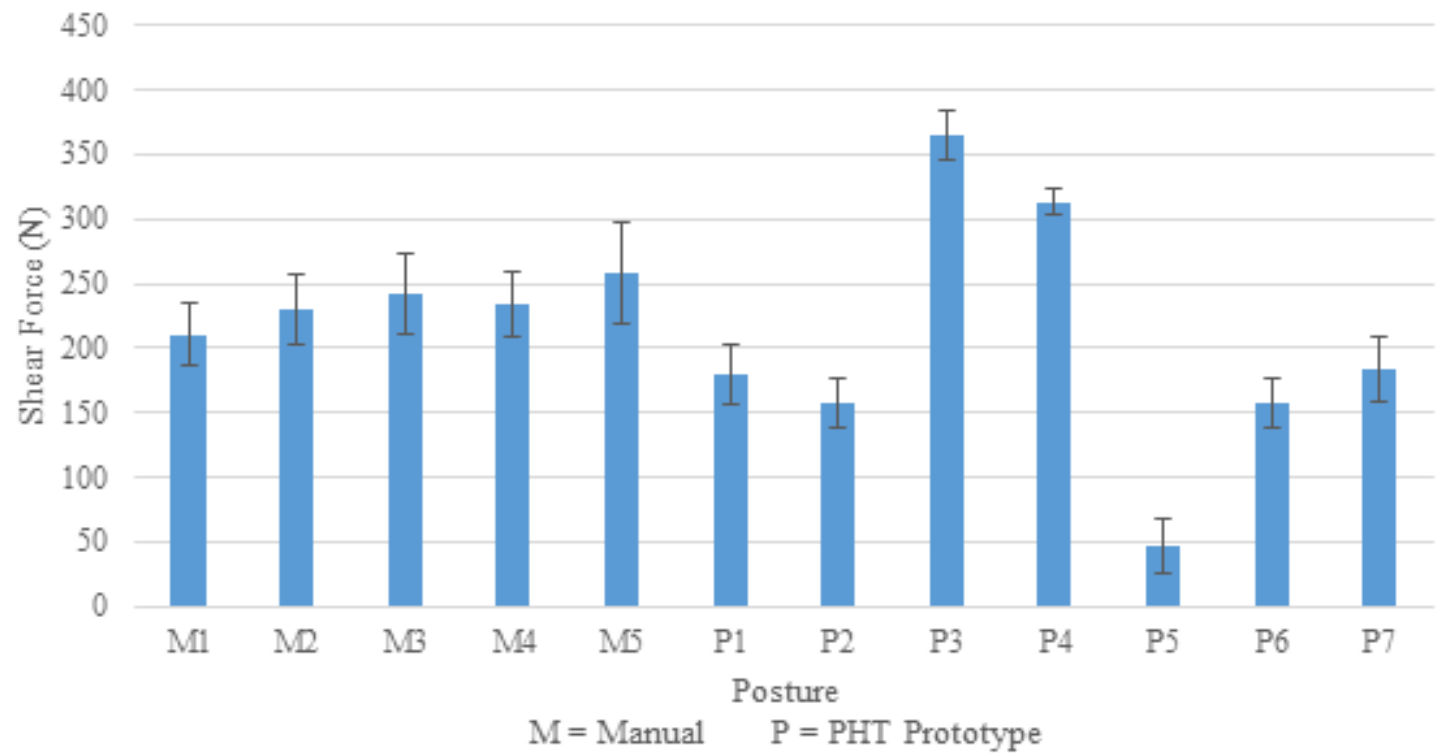

Figure 4 Shear force (mean and standard deviation)

\subsection{REBA and RULA scores}

Based on the REBA method, the scores for the nurses' postures during the manual handling varied between 5 and nearly 9 (Figure 5). Substantially lower scores were observed when the nurses used the prototype (postural scores of less than 3 ). 


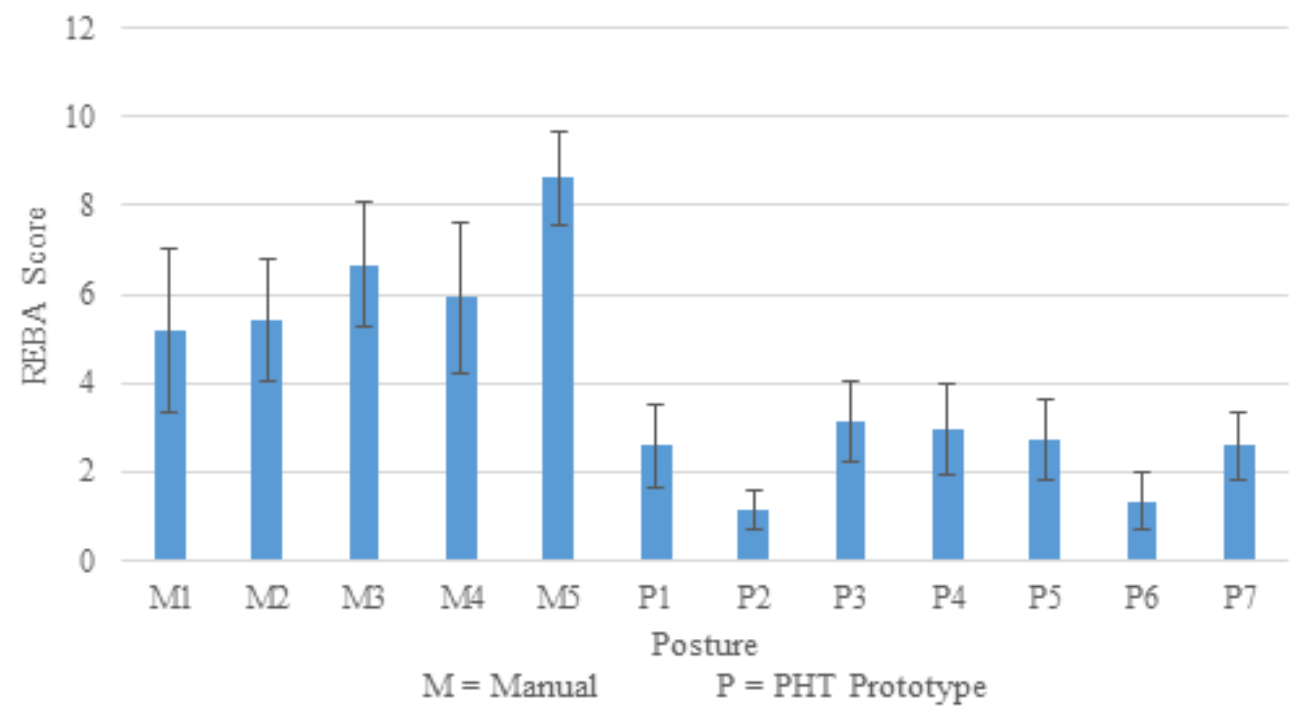

Figure 5 REBA scores (mean and standard deviation)

The postural scores based on the RULA method demonstrated similar results (Figure 6). Scores of 4 to around 7 were observed during manual handling. In contrast, lower scores (between 3 and 4) were found when handling the patient using the PHT prototype.

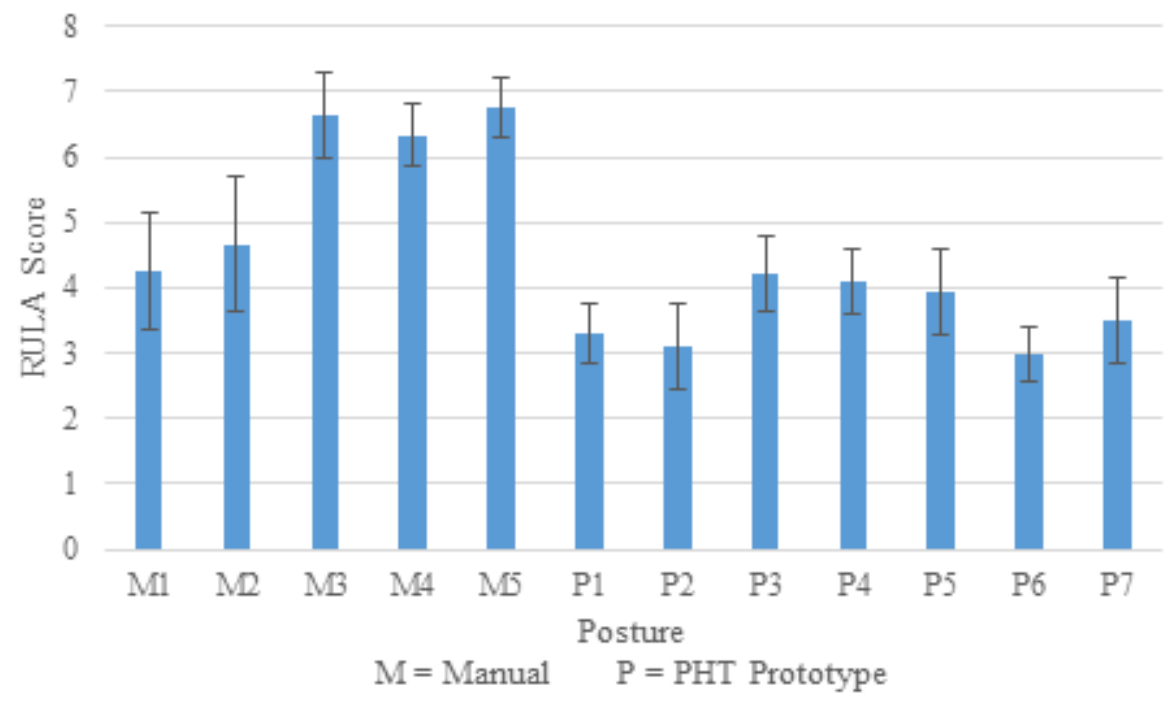

Figure 6 RULA scores (mean and standard deviation)

\subsection{Borg Scale}

The amount of force exerted by the 12 nurse participants was quantified utilizing the Borg Scale. Ranging between 0 and 10, the average rating for the manual handling condition was 6.3. When using the PHT prototype, the average rating was 4.1 (a $35 \%$ reduction compared to the manual handling condition).

\section{Discussion}

The main objective of this research was to evaluate whether the PHT prototype developed locally could indeed be beneficial for nurses and caregivers during patient- 
handling activities. The findings from this research clearly demonstrated that the prototype helped reduce loads on the lower back and improved postural scores. The nurses reported that the PHT reduced the amount of effort exerted during simulated patient handling activities. Across all measures, there was a reduction in ergonomic risk of roughly $30 \%$ to $70 \%$. These results suggest that use of the PHT could potentially ameliorate the musculoskeletal problems experienced by nurses and caregivers in hospitals and other medical settings.

One of the important findings from this investigation is the fact that there was a substantial $(\sim 40 \%)$ reduction in compression force at the (lower back) L5/S1 joint. It has been widely accepted that the compression force at this lumbar joint is strongly associated with the risk of lower back pain, in particular, and of work-related musculoskeletal disorders in general. In this study, the greatest load with the manual condition occurred when positioning the patient onto or away from the wheelchair. Not only was the trunk in the most unnatural (bent) position, it was also necessary to bear the load on one side (on the side where the patient was). In contrast, the use of the PHT eliminated the need to lift the patient from one side, and removed the need to bend in an awkward position that resulted in a compression force at the lumbar joint (Zhuang et al., 1999).

Although the compression force data were improved, using the PHT did require the nurse to exert additional effort during the pushing or pulling of the apparatus. Two additional key postures had to be adopted as compared with the manual handling. These activities created a slightly greater (but less than 30\%) shear force at the L5/S1 joint, as compared to the maximum force that occurred here during the manual handling of the patient. The maximum force when using the PHT was, however, relatively small and was within commonly accepted biomechanical limits. It is noteworthy that the position of the PHT handles relative to the nurse's hand had a considerable effect on the shear lumbar force (Knapik and Marras, 2009). The design of the prototype had not taken into account the positioning of the handles on the PHT. Refinements need to be made to the design by considering the anthropometric data (e.g., the standing elbow height) of the nurses. Additional consideration may also be required regarding the design (type and size) of the casters. A variety of casters are available on the market, but their effects (i.e., increased or reduced friction) on the amount of push/pull force exerted were not investigated.

In this evaluation, the RULA and REBA methods were utilized as means for quantifying the working postures during manual handling as well as when handling the patient using the PHT. Both approaches resulted in high scores, demonstrating high ergonomic risks. In fact, the high scores strongly suggest the need for immediate ergonomic intervention in these patient handling tasks. The use of the PHT as a mechanical aid would certainly reduce these scores, introducing better, more acceptable working postures. The findings of this study show score reductions of up to $62 \%$, which are possibly enough to justify use of the PHT. As previously noted, the use of this mechanical aid does require the nurses to exert push and pull forces. Such exertions would not be captured in the postural scores. The 3DSSPP analysis or the rating of perceived exertion by using Borg's Ratings (see below) should, therefore, be considered.

In this study, the Borg's Ratings were employed to facilitate the nurses in quantifying the amount of effort exerted while handling the patient. The nurse participants valued the use of the technology, and had not been aware that such technology existed. While no data were formally collected, they reported that the PHT seemed to ease their job. Ratings provided by the nurse participants indicated the benefits of the PHT during the simulated patient-handling activities. An average reduction of nearly $35 \%$ was found when they used the PHT prototype. The findings of this study indicate that, subjectively, the nurses felt that 
the aid helped them to perform their task, and reduced the extent of the workload. These findings correspond to the benefits indicated by the 3DSSPP analysis and the REBA/RULA methods.

This research was not without its limitations, and a couple of issues need to be addressed. From the methodological point of view, this study used only a digital camera with markers attached to the approximate body joints. This procedure could have misrepresented the angles between body segments, and might affect the biomechanical and postural analyses. State of the art technology, such as 3D infrared cameras and a force plate (for gait and postural analysis), would be much more reliable in providing angle and force data (Dutta et al., 2011b). However, it was anticipated that the system used in this study would result in only minor angle data deviations. However, ideally, future studies should utilize such advanced technology. Lastly, this study did not take into account the amount of time that the nurses spent using the PHT. It was obvious that using the technology would result in longer handling time. This is an issue that would need to be carefully examined before introducing the PHT into real medical settings. It is not uncommon for medical personnel to move patients quickly under emergency conditions, and, in such circumstances, the use of a transfer aid might not be entirely beneficial.

Despite these limitations, this study clearly demonstrated the substantial benefits of using a mechanical aid (i.e., the PHT) during patient-handling activities. Such technology could greatly reduce the ergonomic risks, and potentially ameliorate the musculoskeletal problems frequently reported by nurses and caregivers. Further work is needed to ensure that the PHT prototype is fully functional in real medical settings, and that the technology is acceptable from the nurses' and caregivers' points of view.

\section{Conclusions}

This study was conducted based on the premise that nurses and caregivers suffer musculoskeletal problems as a result of their manual patient-handling activities. A mechanical aid (i.e., the PHT) had been designed and manufactured previously by the researchers, but it had not yet been evaluated from the biomechanical perspective. This study demonstrated that the use of this technology was ergonomically better than manual handling. The PHT greatly reduced the spinal load (at the L5/S1 joint), improved work postures, and minimized the amount of physical effort during patient handling tasks. Further research is needed to refine the design. It is expected that this technology will help reduce musculoskeletal problems among hospital nurses and caregivers in general, and in particular, among those with regular duties in handling patients.

\section{Acknowledgments}

This research was made possible by a grant provided by the Indonesian Ministry of Research, Technology, and Higher Education (Grant\#: 255/SP2H/LT/DRPM/II/2016).

\section{References}

Alamgir, H., Li, O.W., Yu, S., Gorman, E., Fast, C., Kidd, C., 2008. Evaluation of Ceiling Lifts: Transfer Time, Patient Comfort, and Staff Perceptions. Journal of Injury, Volume 40(9), pp. 987-992

Batubara, H., Dharmastiti, R., 2017. Redesign of Liquid Aluminum Pouring Tool based on Participatory Ergonomics to Improve Productivity, Workload, and Musculoskeletal Disorders. International Journal of Technology, Volume 8(2), pp. 352-361 
Borg, G., 1990. Psychophysical Scaling with Applications in Physical Work and the Perception of Exertion. Scandinavian Journal of Work, Environment \& Health, Volume 16(1), pp. 55-58

Dutta, T., Holliday, P.J., Gorski, S.M., Baharvandy, M.S., Fernie G.R., 2011a. A Biomechanical Assessment of Floor and Overhead Lifts using One or Two Caregivers for Patient Transfer. Journal of Applied Ergonomics, Volume 43(3), pp. 521-531

Dutta, T., Holliday, P.J., Gorski, S.M., Baharvandy, M.S., Fernie G.R., 2011b. The Effects of Caregiver Experience on Low Back Loads during Floor and Overhead Lift Maneuvering Activities. International Journal of Industrial Ergonomics, Volume 41(6), pp. 653-660

Hignett, S., McAtamney, L., 2000. Rapid Entire Body Assessment (REBA). Journal of Applied Ergonomics, Volume 31(2), pp. 201-205

Hodder, J.N., MacKinnon, S.N., Ralhan, A., Keir, P.J., 2010. Effects of Training and Experience on Patient Transfer Biomechanics. International Journal of Industrial Ergonomics, Volume 40(3), pp. 282-288

Iridiastadi, H., Anggawisnu, B., Didin, F.S., Yamin, P.A.R., 2019. The Prevalence of Musculoskeletal Complaints Among Hospital Nurses and Nursing Home Caregivers in Indonesia. International Journal of Technology, Volume 10(4), pp. 854-861

Knapik, G.G., Marras, W.S., 2009. Spine Loading at Different Lumbar Levels during Pushing and Pulling. Journal of Ergonomics, Volume 52(1), pp. 60-70

Maxwell, S.E., Delaney, H.D., 2004. Designing Experiments and Analyzing Data. $2^{\text {nd }}$ Ed. London: Lawrence Erlbaum Associates Publishers

McAtamney, L., Corlett, E.T., 1993. RULA: A Survey Method for the Investigation of Workrelated Upper Limb Disorders. Journal of Applied Ergonomics, Volume 24(2), pp. 91-99

McGuigan, 1990. Experimental Psychology - Methods of Research. New Jersey: Prentice Hall

Nelson, A., Matz, M., Chen, F., Siddharthan, K., Loyd, J., Fagala, G., 2006. Development and Evaluation of Multifaceted Ergonomics Program to Prevent Injuries Associated with Patient Handling Tasks. International Journal of Nursing Studies, Volume 43, pp. 717733

Nelson, A.L., Collins, J., Knibbe, H., Cookson, K., de Castro, A.B., Whipple, K.L., 2007. Safer Patient Handling. Journal of Nursing Management, Volume 38(3), pp. 26-32

Ribeiro, T., Serranhiera, F., Loureiro, H., 2017. Work Related Musculoskeletal Disorders in Primary Health Care Nurses. Applied Nursing Research, Volume 33, pp. 72-77

US Bureau of Labor Statistics, 2008. Fatal Occupational Injuries and Nonfatal Occupational Injuries and Illnesses. Report 1028. Washington DC: US Department of Labor

Waters, T.R., Nelson, A., Proctor, C., 2007. Patient Handling Tasks with High Risk for Musculoskeletal Disorders in Critical Care. Critical Care Nursing Clinics of North America, Volume 19(2), pp. 131-143

Yuhaniz, H., Seraila, A., Karim, S.R.A., Muhammed, S., Saleh, A.H., 2017. Evaluation of Children's Anthropometric Measures in Rural and Urban Areas for Ergonomic Application. International Journal of Technology, Volume 8(2), pp. 230-237

Zhuang, Z., Stobbe, T.J., Hsiao, H., Collins, J.W., Hobbs, G.R., 1999. Biomechanical Evaluation of Assistive Devices for Transferring Residents. Journal of Applied Ergonomics, Volume 30(4), pp. 285-294 